\title{
RELACIÓN ENTRE EL TAMAÑO DEL HOSPEDADOR Y LA INTENSIDAD DE INFECCION DE UN ALGA EPIBIONTE
}

\author{
Carmen Pérez Martínez y José Barea Arco \\ Instituto del Agua, Ramón y Cajal 4, Universidad de Granada, 18071-Granada
}

\begin{abstract}
RESUMEN
El alga epibionte Korshikoviella gracilipes se adhiere al zooplancton de la laguna de Rio Seco (Sierra Nevada). Su principal hospedador es el cladócero Daphniu pulicaria, presentando este taxon valores altos de prevalencia e intensidad de infeccidn por el epibionte. Nuestros resultados muestran que no existe relación entre el tamaño de los individuos hospedadores y la prevalencia e intensidad de infeccidn de los mismos. La ausencia de relacidn puede deberse al incremento de la tasa de encuentro entre epibionte y hospedador como consecuencia de la estrecha asociacidn espacial entre ambas especies en la laguna de Rio Seco.
\end{abstract}

Palabras clave: alga epizoica, Daphnia, tamaño hospedador, intensidad infeccion, prevalencia

\begin{abstract}
The algal epibiont Korshikoviella gracilipes attaches to zooplankton species in Lake Rio Seco (Sierra Nevada). Daphnia pulicaria is the main host of the epibiont, showing the highest values of prevalence and burden. Our results show there is no relation between host size, prevalence and burden. Thisfact could be explained by the increased encounter rute between epibiont and host becuse of the close coincidence of patches of both organisms in this lake.
\end{abstract}

Keywords: algal epibiont, Daphnia, host size, burden, prevulence

\section{INTRODUCCIÓN}

Los epibiontes son organismos que viven la mayor parte de su ciclo de vida adheridos a otro organismo, el hospedador. En su ciclo de vida presentan una fase de dispersion, de vida libre, y una fase de crecimiento y reproduccion, adherida a un sustrato. Los epibiontes sobre crustaceos zooplanctonicos han merecido considerable atención recientemente (Allen et al., 1993; Chiavelli et al., 1993; Threlkeld et al., 1993; Threlkeld \& Willey ,1993; Weissmann et al., 1993; AlDhaheri \& Willey, 1996; Carman \& Dobbs, 1997, Polz et al., 1998). Los crustaceos mudan su exoesqueleto regularmente, de modo que la fase de vida fija y el periodo reproductor de los epibiontes adheridos a su exoesqueleto estan limitados al periodo entre mudas del hospedador. Cuando se produce la muda los epibiontes se desprenden junto con el viejo caparazón. Deben abandonar entonces el mismo y encontrar un nuevo sustrato al que adherirse. Por tanto, el éxito de un epibionte sobre crustaceos depende en gran medida de un eficiente mecanismo de colonizacion y desarrollo sobre el hospedador. Uno de los factores que pueden afectar a la tasa de colonización del epibionte es el tamaiio del hospedador. Por ejemplo, el tamaiio del hospedador influye sobre la tasa de encuentro entre epibionte y hospedador y sobre el crecimiento del epibionte sobre el hospedador a través de la duración del periodo entre mudas (Allen et al., 1993; Threlkeld et al., 1993; Gaiser \& Bachmann, 1993; Stirnadel \& Ebert, 1997).

Korshikoviella gracilipes es un alga verde epibionte que se adhiere a las especies zooplanctónicas de la laguna de Rio Seco (Sierra Nevada). Su ciclo de vida se completa unicamente sobre el 
cladócero Daphniu pulicaria (Sánchez Castillo, 1987). Las densidades de D. pulicaria y el epibionte se relacionan espacial y temporalmente en la laguna y este taxon presenta la mayor intensidad de infección por el epibionte (Barea-Arco et al., 2001). El mecanismo de colonización del epibionte parece ser muy eficiente sobre D. pulicaria. Por ello resulta de interés conocer los factores que influyen sobre la tasa de colonización del epibionte sobre D. pulicaria. En este trabajo se analiza la relación existente entre el tamaiio de los individuos de D. pulicuria y la presencia y el grado de infección de $K$. gracilipes.

\section{MATERIAL Y MÉTODOS}

\section{Sistema estudiado}

Rio Seco es una pequeña laguna $\left(1920 \mathrm{~m}^{2}\right.$, $\mathrm{z}_{\max }=2.90 \mathrm{~m}$ ) situada a 3040 metros s.n.m. en la cordillera de Sierra Nevada (Granada). La laguna permanece helada desde Noviembre hasta Junio aproximadamente, es oligo-mesotrófica y presenta visibilidad total del disco de Secchi. La comunidad planctónica es muy simple. El fitoplancton esta compuesto por unas 10 especies nanoplanctónicas, siendo las dominantes Chromulina nevadensis , Ochromonas sp., Dictyosphaerium chlorelloides y las zoosporas del alga epizoica $K$. grucilipes. Las especies zooplanctónicas dominantes son el calanoide Mixodiaptomus laciniatus y el cladócero Daphnia pulicaria.

\section{Ciclo de vida de K. gracilipes:}

$K$. gracilipes presenta un ciclo de vida complejo (Sánchez-Castillo, 1987). El ciclo de vida asexual comprende cinco formas de vida: zoospora, clorangioide, anluroide, adulto y ciste.

Las zoospores son células biflageladas planctónicas (largo $=7.98 \pm 1.30 \mu \mathrm{m}(\mathrm{x} \pm$ s.d. $)$, ancho $=3.90 \pm 0.75 \mu \mathrm{m}$; biovolumēn $=69.00 \pm$ $37.31 \mu \mathrm{m}^{3}, \mathrm{n}=1495$ ). La fase de zoospora es la fase dispersiva del epibionte. Cuando una zoospora encuentra un organismo sustrato se adhiere por su región apical y pierde el flagelo durante el proceso, transformandose en clorangioide. Los clorangioides se transforman en ankiroides mediante división transversal. Los ankiroides son entonces organismos bicelulares que derivan en adultos por alargamiento celular y sucesivas divisiones transversales. La fase adulta $(\operatorname{largo}=140.69 \pm 64.65 \mu \mathrm{m}$, ancho $=10.14 \pm 2,85$ $\mu \mathrm{m}$; biovolumen $=14447.70 \mu \mathrm{m}^{3}, \mathrm{n}=543$ ) es la fase reproductiva del ciclo de vida de $K$. gracilipes. Cada adulto puede producir zoosporas por mitosis y, bajo ciertas condiciones, cistes. Los cistes son formas de resistencia que sobreviven al invierno.

Los clorangioides se adhieren fundamentalmente sobre $M$. laciniatus y $D$. pulicaria. Los ankiroides y adultos se encuentran casi exclusivamente sobre el aparato filtrador de $D$. pulicuria, de modo que $K$. gracilipes unicamente completa su ciclo de vida sobre D. pulicaria.

\section{Recolección y análisis de las muestras}

Las muestras de plancton se tomaron en cinco puntos (1-5) de la laguna desde Julio a Noviembre de 1996. El área del lago se dividió en cinco zonas y se tom6 una muestra aleatoriamente en cada una de las zonas. El fitoplancton y zooplancton se recolectó de una muestra homogénea de 10 litros de agua bombeada con una bomba eléctrica desde 0.5 metros por encima del fondo de la laguna en cada punto de muestreo. Las muestras de fitoplancton $(100 \mathrm{ml})$ se fijaron inmediatamente con Lugol. El zooplancton se filtró por una red de $40 \mu \mathrm{m}$ de tamaiio de poro y se fijó con formol al 4\% azucarado. El recuento de especies fito y zooplanctónicas se realizó con un microscopio invertido Leitz segun la técnica de Utermohl.

Para el análisis de la relación entre tamaiio de D. pulicaria e infección por $K$. gracilipes se midieron y examinaron los individuos de D.pulicuria de cada punto y dia de muestreo. El tamaiio se estimó mediante la medida de la longitud del cuerpo entre la base de la espina y la parte superior del ojo. En muestras con menos de 100individuos se examinaron todos ellos $\mathbf{y}$ en muestras mayores se analizaron al menos 100 individuos. La prevalencia del epibionte, definida como el porcentaje de individuos con epibiontes, se calculó para cada muestra. La intensidad de infec- 
cion, definida como el numero de epibiontes sobre un organismo sustrato, se midi6 siguiendo una escala visual arbitraria del 0 al 5 , con $0=$ ausencia de infección y $5=$ maxima infección (Barea-Arco et al., 2001). En el aiio 1996 la prevalencia y densidad de infección por adultos de $K$. gracilipes fue extremadamente baja. Por ello en este trabajo nos referimos unicamente al estudio de la infeccion por clorangioides.

La tasa de colonización por un epibionte sobre un hospedador puede depender considerablemente de las probabilidades de encuentro entre ambos, lo cual depende a su vez de la densidad de ambas poblaciones (Threlkeld et al., 1993). Por esta razón, el analisis de la relación entre tamaiio del hospedador e intensidad de infeccion (prevalencia y grado de infección) se realizo para cada muestra individual (cada punto y dia de muestreo) y no para el conjunto de todas ellas con el fin de asegurar que todos los individuos analizados se encuentren en las mismas condiciones.

\section{RESULTADOS}

Los primeros individuos de $D$. pulicaria se observaron a principios de Agosto (Fig. 1). La densidad poblacional present6 valores bajos durante todo el mes; aumento considerablemente a partir de Septiembre y mantuvo valores altos hasta el final del periodo de muestreo. El tamaiio medio de los individuos aumentó al avanzar el periodo libre de hielo, probablemente debido a que el incremento de la densidad poblacional se realiza a través de juveniles procedentes de efipios al principio del periodo. La prevalencia media y la intensidad media de infección muestran una correlación positiva con la densidad de D. pulicaria (Fig. 1; correlación de Pearson; prevalencia, $r=$ $0.84, p=0.001 ;$ intensidad de infección, $r=0.78$, $\mathrm{p}=0.003$ ).

El análisis entre el tamaiio y prevalencia e intensidad de infección se ha realizado sobre las muestras del 3 de Septiembre en adelante para evitar los primeros días de muestreo, donde la mayoría de los individuos son juveniles y el numero de individuos y la intensidad de la infec-
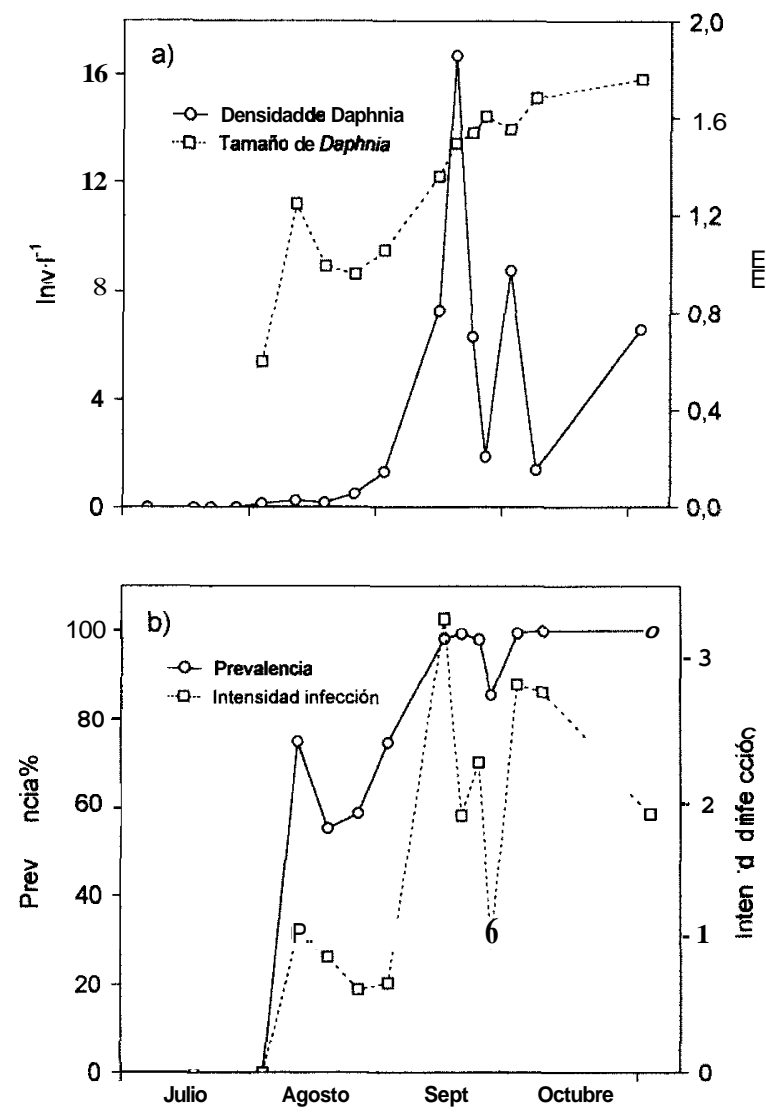

Fig. 1. a) Densidad y tamaiio y b) prevalencia e intensidad de infección de los individuos de D. pulicaria en el periodo de muestreo de 1996. Se presentan los valores medios de los cinco puntos de muestreo. a) abundance and bodysize, and b) prevalence and burden of Daphnia pulicaria individuals during the sampling period of 1996. Mean values for thefive sampling sites are shown.

ción es extremadamente baja, presentando todos los individuos infectados un grado 1 de infeccion. El rango de tamaiio del total de los individuos analizados osciló entre 0.689 y $2.99 \mathrm{~mm}$ y el tamaiio medio fue de $1.507 \pm 0.333$ ( $\mathrm{x} \pm$ s.d., $\mathrm{n}=$ 933). El analisis sobre los individuos de cada punto y dia de muestreo se realizó sobre aquellas muestras que presentaban más de 5 individuos analizados y mas de 2 niveles de intensidad de infeccion, resultando un total de 20 muestras. El ANOVA de una via realizado para cada muestra 
revel6 que no existen diferencias significativas de tamafio entre individuos infectados y no infectados en cada una de las 20 muestras (valores de $p$ $>0.05$ para cada muestra). La relacion entre el tamaiio de los individuos y grado de infeccion solamente resultó significativa para el punto $1 \mathrm{del}$ 16 de Septiembre (correlación de Spearman; $r=$ $0.404, \mathrm{p}<0.001, \mathrm{n}=162$ ) y para el punto $1 \mathrm{del}$ 24 de Septiembre (correlacion de Spearman; $r=$ $0.272, \mathrm{p}=0.015, \mathrm{n}=80$ ).

\section{DISCUSIÓN}

La relacion entre tamafio del hospedador e infeccion presenta amplia variabilidad en los estudios de epibiontes sobre crustaceos (ver tabla 1 de Threlkeld et al., 1993). Algunos autores encuentran una relacion positiva (Holland \& Hergenrader, 1981; Møhlenberg \& Kaas, 1990) mientras que otros como Weissmann et al. (1993) y Kankaala \& Eloranta (1987) no encuentran relacion entre la carga de ciliados epibiontes y el tamafio de sus hospedadores. Esta relacion puede incluso variar para un mismo sistema y hospedador respecto a distintas especies de epibiontes. Este es el caso de Chlorangiella sp. y Colacium sp. cuya prevalencia se relaciona positivamente con el tamafio de $D$. pulex y $D$. rosea mientras que la relacion de Carchesium sp. es negativa con la primera y neutra con la segunda (Threlkeld \& Willey, 1993).

Nuestros resultados muestran que no existe relacion entre el tamafio de los individuos y la presencia o ausencia de infeccion, resultado esperable si tenemos en cuenta que el valor de la prevalencia de la infeccion en las muestras analizadas esta por encima del $75 \%$ (Fig. 1). Además, nuestros resultados parecen indicar igualmente que no existe relacion entre el tamaiio de D. pulicaria y el número de epibiontes adheridos sobre su caparazon, aunque la relacion es positiva para 2 de las 20 muestras analizadas. De estos hechos pueden obtenerse algunas conclusiones sobre el mecanismo de colonización de $K$. gracilipes sobre D. pulicaria. Los altos valores de prevalencia sugieren que la probabilidad de infeccion es extremadamente alta para los individuos de $D$. pulicaria. Esta probabilidad depende en gran medida de la tasa de encuentro entre hospedador y epibionte (Chiavelli et al., 1993; Threlkeld et al., 1993; Gaiser \& Bachmann, 1993), probablemente facilitada por la asociación espacial que presentan ambas especies en la laguna de Rio Seco, donde se observan agregados coincidentes de las densidades de las dos especies (Barea-Arco et al., 2001). La colonizacion dentro de los agregados de Daphnia y zoosporas debe ser continua y muy rapida e independiente del tamaiio de los hospedadores. Continuamente se desprenden epibiontes de individuos en proceso de muda y éstos encuentran facilmente un nuevo individuo para colonizar dentro del agregado.

La relacion positiva entre prevalencia y tamafio puede deberse al incremento de las tasas de filtración en individuos de mayor tamaño y, consecuentemente, de las probabilidades de contacto con el epibionte (Stirnadel \& Ebert, 1997). Por otro lado, las variaciones en tamaiio pueden afectar a la susceptibilidad de infeccion dado que la probabilidad de encuentro incrementara al aumentar el area superficial del hospedador (Gaiser \& Bachmann, 1993). Es probable que la relacion tamaiio hospedador-intensidad de infeccion muestre un comportamiento densidaddependiente de modo que se manifieste en condiciones de baja densidad de una o ambas especies mientras que la relacion se debilita al incrementar la densidad de las especies interactuantes. Este seria el caso del sistema $K$. gracilipes- $D$. pulicaria en Rio Seco, donde la probabilidad de encuentro entre ambas especies dentro de los agregados es tan alta que podría ser independiente del potencial incremento de la misma debido al tamaiio del hospedador o a las corrientes de agua producidas por el mismo.

La colonización puede verse facilitada, además, por la difusión de sustancias químicas atrayentes para el epibionte por parte de Daphnia, tal como sugiere Al-Dhaheri \& Willey (1996). Es también posible que el individuo en proceso de muda reciba los epibiontes desprendidos de su antigua muda, con lo que el tamafio del 
hospedador no influiria en la colonización. De acuerdo con esta ultima hipdtesis, hemos observado en el laboratorio clorangioides adheridos al nuevo exoesqueleto de individuos que aun no habian completado el proceso de muda.

La intensidad de infeccidn depende de la tasa de nuevas colonizaciones y, además, de la tasa de crecimiento del epibionte sobre el hospedador, afectada por la duracidn del periodo entre mudas. Los individuos analizados en este estudio presentan un amplio rango de tamaño pero son en su mayoria adultos y probablemente muestren pocas diferencias de duracidn del periodo entre mudas. Este hecho pudo afectar a la ausencia de relacion entre ambos parametros. Por ejemplo, Allen et al. (1993) encuentra que la intensidad de infeccidn por diatomeas es mayor en adultos que en juveniles de Daphnia, atribuyendo este hecho a la diferencia de la duracidn del periodo entre mudas entre ambos estadios. Esta hipdtesis resulta difícil comprobar en Rio Seco, donde D. pulicaria se reproduce mayoritariamente por huevos efipiales.

En resumen, en nuestro estudio no se observa una relación clara entre tamaño del hospedador e infeccidn por $K$. gracilipes. La ausencia de esta relacion puede deberse a diversas causas entre las que se destaca la facilitación de la colonización por la estrecha asociacion espacial entre ambas especies en la laguna de Rio Seco.

\section{AGRADECIMIENTOS}

Este trabajo ha sido parcialmente financiado por los proyectos CICYT AMB94-0021 y CICYT AMB99-0541.

\section{REFERENCIAS}

AL-DHAHERI, R. S. \& R.L. WILLEY. 1996. Colonization and reproduction of the epibiotic flagellate Colacium vesiculosum (Euglenophyceae) on Daphnia pulex. J. Phycol., 32, 770-774.

ALLEN, Y. C., B. T. DE STASIO \& C. W. RAMCHARAN. 1993. Individual and population level consequences of an algal epibiont on Daphnia. Limnol. Oceanogr., 38(3), 592-601.

BAREA ARCO, J., c. PÉREZ MARTÍNEZ \& R. MORALES BAQUERO. 2001. Evidence of a mutualistic relationship between an algol epibiant and its host, Daphnia publicaria. Limnol. Oceanogr, 46:871-88I.

CARMAN, K. R. \& F.C. DOBBS. 1997. Epibiotic microorganisms on copepods and other marine crustaceans. Microsc. Res. Techniq., 37, 116-135.

CHIAVELLI, D. A., E. L. MILLS \& S.T. THRELKELD. 1993. Host preference, seasonality, and community interactions of zooplankton epibionts. Limnol. Oceunogr, 38(3), 574-583.

GAISER, E. E. \& R.W. BACHMANN. 1993. The ecology and taxonomy of epizoic diatoms on Cladocera. Limnol. Oceanogr., 38(3), 628-637.

HOLLAND, R. S. \& G.L. HERGENRADER. 1981. Bacterial epibionts of diaptomid copepods. Trans. Am. Microsc. Soc., 100, 56-65.

KANKAALA, P. \& P. ELORANTA. 1987. Epizooic ciliates (Vorticella sp.) compete for food with their host Daphnia longispina in a small polyhumic lake. Oecologia, 73, 203-206.

MOHLENBERG, F. \& H. KAAS. 1990. Colacium vesiculosum Ehrenberg (Euglenophyceae) infestation of planktonic copepods in the western Baltic. Ophelia, 31, 125-132.

POLZ, M. F., J.J. ROBINSON, C.M. CAVANAUGH \& C.L. VAN DOVER. 1998. Trophic ecology of massive shrimp aggregations at a Mid-Atlantic Ridge hydrothermal vent site. Limnol. Oceanogr., 43, 1631-1638.

SANCHEZ-CASTILLO, P. M. 1987. Estudio del ciclo biológico de Korshikoviella gracilipes (Lambert) Silva (Chlorococcales, Chlorophyta). Phycologia, 26(4), 496-500.

STIRNADEL, H. A. \& D. EBERT. 1997. Prevalence, host specificity and impact on host fecundity of microparasites and epibionts in three sympatric Daphnia species. J. Anim. Ecol., 66, 212-222. 
THRELKELD, S. T. \& R.L. WILLEY. 1993. Colonization, interaction, and organization of cladoceran epibiont communities. Limnol. Oceanogr, 38(3), 584-591.

THRELKELD, S. T., D.A. CHIAVELLI \& R.L. WILLEY. 1993. The organization of zooplankton epibiont communities. Trends Ecol. Evol., 8(9), 317-321.
WEISSMAN, P., D.J. LONSDALE. \& J. YEN. 1993.

The effect of peritrich ciliates on the production of Acartia hundsonica in Long Island Sound. Limnol. Oceanogr, 38(3), 613-622. 